\title{
A Multi-phase hp-adaptive Radau Pseudospectral Method and its Application to Formation Optimal Control
}

\author{
Fang Wang ${ }^{1,2, a}$, Naigang Cui ${ }^{1, b}$ \\ ${ }^{1}$ School of Astronautics, Harbin Institute of Technology, Harbin 150001, China; \\ ${ }^{2}$ Beijing Electro-mechanical Engineering Institute, Beijing 100074, China. \\ awangfang_hit@163.com, ${ }^{\text {b }}$ cui_naigang@163.com
}

Keywords: Formation Combat Integration, Energy Optimal Control, Multi-phase, hp-adaptive.

\begin{abstract}
In view of the formation combat optimal control problem, integrated rally - keep - attack formation control algorithm is put forward to minimize the overall energy. Equations of state have been established while considering the dynamic characteristics of members, the delay of control system, and target motion features. Through decomposing the task of formation combat, three subtasks optimal control model are established respectively with each subtask characteristic. Then the optimal control model of formation combat system is formed. To resolve the different characteristics of control restrictions in each phase, this paper proposes multi-phase hp-adaptive Radau pseudospectral method to solve the energy optimal control problem and to realize autonomous switching on three subtasks. It is shown from simulation results that optimal control instruction which meet the demand of the task can be quickly obtained using this method and $7 \%$ of energy is saved.
\end{abstract}

\section{Introduction}

Aircraft formation flying technology has been called "future space missions to science and technology" by the U.S. Air Force Research Lab and NASA, and has been widely recognized because of its good fault tolerance, robustness, low cost, and multitasking ability. Many scholars carry out the relevant research work and have achieved some results on the applications of unmanned aerial vehicle $^{[1]}$, robot $^{[2]}$ and satellite ${ }^{[3]}$.

Many researches ${ }^{[4-15]}$ focused on the formation structure and formation control can be summarized as follows: leader-follower ${ }^{[6,7]}$, based behavior ${ }^{[8,9]}$, virtual structure ${ }^{[10,11]}$, and graph theory ${ }^{[12,13]}$. Leader-follower is popular with the prominent advantages that through designing the leader's behavior to control the whole formation. It is important to study on integrated optimal control problem of formation rally, formation keeping and coordinated attack, but few people have made any monographic study ever before. An integration optimal control algorithm of a leader-follower mode is put forward in this article in order to minimize the energy of whole process, and many factors such as anti-collision, formation parameters, condition of association, missile control limit, and hit precision are considered.

\section{States model of formation combat system}

Formation combat system is composed of the members, command and control system, detecting and tracking system, and fixed/moving target. State models of members and target are focused in this paper, neglecting the influence of other systems.

\subsection{Dynamic Model of the Member}

The dynamic model of member including control delay is given with the assumption that integrated guidance and control method was used in each member while the deflection angle was designed as guidance command. 


$$
\begin{aligned}
& \dot{\mathrm{x}}=V \cos \theta \cos \psi_{c} \\
& \dot{\mathrm{y}}=V \sin \theta \\
& \dot{\mathrm{z}}=-V \cos \theta \sin \psi_{c} \\
& \dot{V}=\left(P-C_{x} q S-m g \sin \theta\right) / m \\
& \dot{\theta}=\left(C_{y}^{\delta y} \delta_{y} q S-m g \cos \theta\right) /(m V) \\
& \dot{\psi}_{c}=-C_{z}^{\delta z} \delta_{z} q S /(m V \cos \theta) \\
& \dot{m}=\dot{m}_{c} \\
& \dot{\delta}_{y}=\frac{\delta_{\text {ycmd }}-\delta_{y}}{T_{s}} \\
& \dot{\delta}_{z}=\frac{\delta_{z c m d}-\delta_{z}}{T_{s}}
\end{aligned}
$$

Eq. (1) is denoted as below: $\quad \dot{X}_{L, F_{i}}=f\left(X_{L, F_{i}}, U_{L, F_{i}}\right)$

Where $X_{L, F_{i}}=\left[\begin{array}{lllllllll}x & y & z & v & \theta & \psi_{c} & m & \delta_{y} & \delta_{z}\end{array}\right]^{T}$ and $U_{L, F_{i}}=\left[\begin{array}{lll}\delta_{y c m d} & \delta_{z c m d}\end{array}\right]^{T}$ mean the state variables and control variables of member.

\subsection{Kinetic model of mobile target}

To reflecting operation capability of formation for maneuvering target, the kinetic model of mobile object is given as follows:

$$
\begin{aligned}
& \dot{\mathrm{x}}_{T}=V_{T} \cos \theta_{T} \cos \psi_{c T} \\
& \dot{\mathrm{y}}_{T}=V_{T} \sin \theta_{T} \\
& \dot{\mathrm{z}}_{T}=-V_{T} \cos \theta_{T} \sin \psi_{c T} \\
& \dot{V}_{T}=a_{x T} \\
& \dot{\theta}_{T}=a_{y T} / V_{T} \\
& \dot{\psi}_{c T}=a_{z T} /\left(V_{T} \cos \theta_{T}\right)
\end{aligned}
$$

where $a_{x T}, a_{y T}$ and $a_{z T}$ are three directions of estimated acceleration in velocity coordinate of target ${ }^{[16]}$. Eq. (3) is shorthand for

$$
\dot{X}_{T}=f_{T}\left(X_{T}\right)
$$

\subsection{Motion model of formation combat system}

Based on the above-mentioned models, motion equation of formation combat system is provided as below:

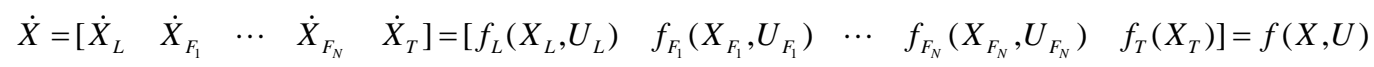

where $N$ is the number of followers; $U$ means control variables of leader and follower:

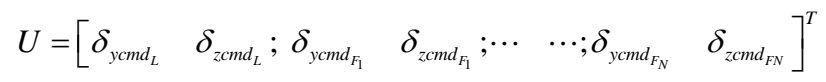

As shown in Eq. (5), motion model of formation combat system becomes more complex and makes the solver jobs harder with the control performance of member and maneuver characteristics of target. But it can truly reflect the movement characteristics of formation combat system, which is necessary for engineering.

\subsection{Relative position between leader and follower}

In order to show the relative position between leader and follower clearly in formation combat, (as shown in Fig. 1), relative location model is given in velocity coordinate of leader:

where

$$
\begin{aligned}
& R_{L F}=\sqrt{X_{r v}^{2}+Y_{r v}^{2}+Z_{r v}^{2}} \\
& \theta_{L F}=\arcsin \left(Y_{r v} / R_{L F}\right) \\
& \psi_{L F}=\arctan \left(-Z_{r v} / X_{r v}\right)
\end{aligned}
$$

$$
\left[\begin{array}{l}
X_{r v} \\
Y_{r v} \\
Z_{r v}
\end{array}\right]=\left[\begin{array}{ccc}
\cos \left(\theta_{L}\right) & \sin \left(\theta_{L}\right) & 0 \\
-\sin \left(\theta_{L}\right) & \cos \left(\theta_{L}\right) & 0 \\
0 & 0 & 1
\end{array}\right]\left[\begin{array}{ccc}
\cos \left(\psi_{c L}\right) & 0 & -\sin \left(\psi_{c L}\right) \\
0 & 1 & 0 \\
\sin \left(\psi_{c L}\right) & 0 & \cos \left(\psi_{c L}\right)
\end{array}\right]\left[\begin{array}{l}
x_{F}-x_{L} \\
y_{F}-y_{L} \\
z_{F}-z_{L}
\end{array}\right]
$$

The relative location model built in velocity coordinate is different from traditional inertial 
coordinate in this paper. It can make the direction of formation being consistent with attack direction of leader at all times and keep the overall performance of formation.

In Fig. 1, XYZ is inertial coordinate and $X_{v} Y_{v} Z_{v}$ means velocity coordinate of leader; $R_{L F}, \theta_{L F}, \psi_{L F}$ are shape parameters.

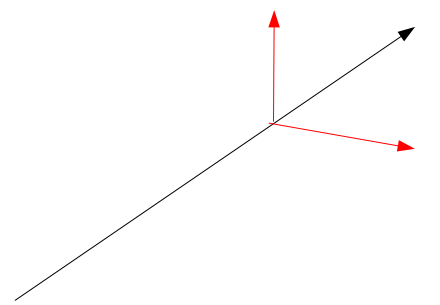

Fig. 1. The relationship between the leader and follower
Fig. 2. The process of formation combat

\section{Optimal Control Model of Formation Combat System}

\subsection{Decomposition of combat mission}

According to the operation task of formation combat the whole process can be divided into three stages: formation rally, formation keeping and coordinated attack.

In Fig. 2, $R_{L F C}, \theta_{L F C}, \psi_{C L F C}$ are the desired structure parameters of formation, and indicate distance, elevation angle and azimuth angle between follower and leader.

\subsection{Optimal control model of formation rally}

In formation rally phase, ${ }^{t \in\left[t_{0}, t_{r}\right]}$, members quickly assemble in the formulated formation with minimized energy consumption19) and the distance between each members should exceeds a safe distance, while allowing to keep communication. At time ${ }^{t_{r}}$, formation errors must meet the requirements.

Optimal control model in formation rally phase is built as follows:

$$
\begin{aligned}
& J^{(1)}=\int_{t_{0}}^{t_{F}}\left[\left(\left|\delta_{y_{L}}\right|^{2}+\left|\delta_{z_{L}}\right|^{2}\right)+\sum_{i=1}^{N}\left(\left|\delta_{y_{F_{i}}}\right|^{2}+\left|\delta_{z_{F_{F}}}\right|^{2}\right)\right] d t \\
& \text { s.t. }^{(1)}\left\{\begin{aligned}
\text { Dynamic : } & \dot{X}=f(X, U) \\
\text { Terminal }: & \left|R_{L F_{i}}\left(t_{r}\right)-R_{L F c}\right| \leq \varepsilon_{R} \\
& \&|| \theta_{L F_{i}}\left(t_{r}\right)\left|-\theta_{L F c}\right| \leq \varepsilon_{\theta_{L F}} \\
& \&|| \psi_{L F_{i}}\left(t_{r}\right)\left|-\psi_{L F c}\right| \leq \varepsilon_{\psi_{L F}} \\
& \&\left|\theta_{F_{i}}\left(t_{r}\right)-\theta_{L}\left(t_{r}\right)\right| \leq \varepsilon_{\theta} \\
& \&\left|\psi_{c F_{i}}\left(t_{r}\right)-\psi_{c L}\left(t_{r}\right)\right| \leq \varepsilon_{\psi_{c}} \\
\text { Process : } & R_{\text {safe }} \leq R_{L F_{i}}(t) \leq R_{\text {commu }} \\
& \& R_{\text {safe }} \leq R_{F_{i} F_{j}}(t) \leq R_{\text {commu }} \\
& \&\left|U_{j}(t)\right| \leq \delta_{\text {max }}, \mathrm{j}=1,2, \cdots, 2 *(\mathrm{~N}+1)
\end{aligned}\right.
\end{aligned}
$$

where $\left[\begin{array}{lllll}\varepsilon_{R} & \varepsilon_{\theta_{L F}} & \varepsilon_{\psi_{L F}} & \varepsilon_{\theta_{L}} & \varepsilon_{\psi_{c L}}\end{array}\right]$ is the formation permissible error, including distance, elevation angle and azimuth angle, path angle and heading angle; $R_{\text {safe }}$ and $R_{\text {commu }}$ are the safe distance and maximized communication distance. 


\subsection{Optimal control model of formation keeping}

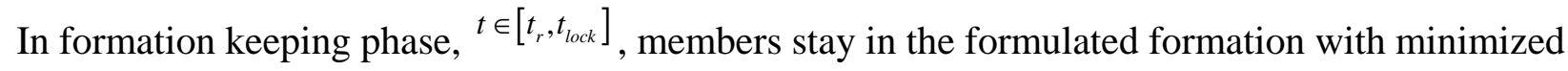
energy consumption. At time ${ }^{t_{\text {lock }}}$, the leader lock onto a target.

Optimal control model in formation keeping phase is built as below:

$$
\begin{aligned}
& J^{(2)}=\int_{t_{\text {tock }}}^{t_{r}}\left[\left(\left|\delta_{y_{L}}\right|^{2}+\left|\delta_{z_{L}}\right|^{2}\right)+\sum_{i=1}^{N}\left(\left|\delta_{y_{F_{i}}}\right|^{2}+\left|\delta_{z_{F_{i}}}\right|^{2}\right)\right] d t \\
& \text { s.t. }^{(2)}\left\{\begin{aligned}
\text { Dynamic : } & \dot{X}=f(X, U) \\
\text { Terminal : } & \left|R_{L T}\left(t_{\text {lock }}\right)-R_{\text {lock }}\right| \leq \varepsilon_{R_{\text {lock }}} \\
\text { Process : } & \&\left|\phi_{L T}\left(t_{\text {lock }}\right)-\phi_{\text {lock }}\right| \leq \varepsilon_{\phi_{\text {lock }}}(t)-R_{L F c} \mid \leq \varepsilon_{R} \\
& \&\left|\theta_{L F_{i}}(t)\right|-\theta_{L F c} \mid \leq \varepsilon_{\theta_{L F}} \\
& \&\left|\psi_{L F_{i}}(t)\right|-\psi_{L F c} \mid \leq \varepsilon_{\psi_{L F}} \\
& \&\left|\theta_{F_{i}}(t)-\theta_{L}(t)\right| \leq \varepsilon_{\theta} \\
& \&\left|\psi_{c F_{i}}(t)-\psi_{c L}(t)\right| \leq \varepsilon_{\psi_{c}} \\
& \&\left|U_{j}(t)\right| \leq \delta_{\text {max }}, \mathrm{j}=1,2, \cdots, 2 *(\mathrm{~N}+1)
\end{aligned}\right.
\end{aligned}
$$
them.

where $R_{\text {lock }}$ and $\phi_{\text {lock }}$ are the effective range and angle, $\varepsilon_{R_{\text {lock }}}$ and ${ }_{\phi_{\text {lock }}}$ means the permissible errors of

\subsection{Optimal control model of coordinated attack}

After lock onto the target, the formation will fly to the object until achieve time/angle coordinated attack $^{[17,18]}$ at time $t_{f}$, when the distance between members and target less than its destructive radius $R_{\text {destroy }}$ and the attack angle $\theta_{\text {expectation }}$ is allowable.

$$
\begin{aligned}
& J^{(3)}=\int_{t_{f}}^{t_{\text {occ }}}\left[\left(\left|\delta_{y_{L}}\right|^{2}+\left|\delta_{z_{L}}\right|^{2}\right)+\sum_{i=1}^{N}\left(\left|\delta_{y_{F_{i}}}\right|^{2}+\left|\delta_{z_{F_{i}}}\right|^{2}\right)\right] d t \\
& \text { s.t. }^{(3)}\left\{\begin{aligned}
\text { Dynamic : } & \dot{X}=f(X, U) \\
\text { Terminal : } & R_{T L}\left(t_{f}\right) \leq R_{\text {destroy }} \\
\& & R_{T F_{i}}\left(t_{f}\right) \leq R_{\text {destory }} \\
& \&\left|\theta_{L}\left(t_{f}\right)-\theta_{\text {expectaion }}\right| \leq \varepsilon_{\theta_{\text {expecation }}} \\
& \&\left|\theta_{F_{i}}\left(t_{f}\right)-\theta_{\text {expectaion }}\right| \leq \varepsilon_{\theta_{\text {expectation }}} \\
\text { Process: } & R_{\text {safe }} \leq R_{L F_{i}}(t) \\
\& & R_{\text {safe }} \leq R_{F_{i} F_{j}}(t), i \neq j \\
\& \mid & \left|U_{j}(t)\right| \leq \delta_{\max }, j=1,2, \cdots, 2 *(\mathrm{~N}+1)
\end{aligned}\right.
\end{aligned}
$$

where $\varepsilon_{\theta_{\text {expectation }}}$ is permissible error of attack angle.

\subsection{Optimal control model of formation combat}

In allusion to the whole action task, the optimal control model of formation combat is given based on the optimal control model of subtask affront mentioned in Eq. (7) Eq. (9).

$$
\begin{aligned}
& \min J=\sum_{p=1}^{3} J^{(p)} \\
& \text { s.t. } \quad \text { s.t. }{ }^{(p)} \\
& X^{(1)}\left(t_{r}^{-}\right)=X^{(2)}\left(t_{r}^{+}\right) \\
& X^{(2)}\left(t_{\text {lock }}^{-}\right)=X^{(3)}\left(t_{\text {lock }}^{+}\right)
\end{aligned}
$$


where $p$ means $p^{\text {th }}$ phase. The restriction functions include two continuous restrictions beside the restrictions of each phase.

\section{Numerical Method}

It is very difficult to solve the optimal control model with nonlinear dynamic equations, and the approximately method are used to translate optimal control problem into nonlinear program problem in a large number of domestic and foreign documents. The state and control variables are closed by polynomial in this approximately method in order to avoid much integral operation to improve the optimization iteration speed ${ }^{[20]}$.

The Radau Pseudospectral Method has been chosen because of its astringency more than Gauss Pseudospectral Method. It needs to set the number of mesh refinement iterations and number of allowable collocation points in a mesh interval in advance, which impact to the precision and speed. And hp-adaptive method has emerged.

The procedure of the hp-adaptive Radau Pseudospectral Method ${ }^{[21]}$ as shown in Fig.3.

In the formation combat optimal control problem, the more followers, the more state variables and restrictions are exist, and the computing speed is more slowly. In order to accelerate the computing speed the multi-phase method is put forwarded. The optimal control problem is divided into three sub optimal control problems according to the subtask phase, and the sub optimal control problems are solved independently by hp-adaptive Radau Pseudospectral Method with state continuous restriction.

Furthermore, using the multi-phase method can optimize the control variables and time nodes $\left(t_{r}, t_{\text {lock }}, t_{f}\right)$ simultaneously, which realizes the transfer of sub phase initiatively. 


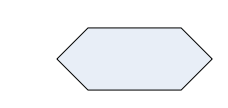

Fig. 3. The procedure of the hp-adaptive RPM

\section{Simulation}

A simulation examination is designed in MATLAB to check up the proposed method for the formation combat optimal control problem.

On the assumption that formation combat system is consist of one leader, one follower and one target, whose attribute parameters and initial values of state are given in Tab. 1 and Tab. 2.

Table 1. Attribute parameters

\begin{tabular}{cc}
\hline Denomination & Value \\
\hline Anticipant distance between leader and follower $R_{L F c}$ & $1000 \mathrm{~m}$ \\
Anticipant elevation angle between leader and follower $\theta_{L F c}$ & $0^{\circ}$ \\
Anticipant azimuth angle between leader and follower $\psi_{L F c}$ & $30^{\circ}$ \\
Allowed error of follower $\varepsilon_{R \theta \psi}$ & {$\left[100 \mathrm{~m} 1^{\circ} 1^{\circ}\right]$} \\
Safe distance between each member $R_{\text {safe }}$ & $300 \mathrm{~m}$ \\
Maximized communicate distance $R_{\text {commu }}$ & $10 \mathrm{~km}$ \\
characteristic area $S$ & $0.2 \mathrm{~m}^{2}$ \\
Time delay $T_{s}$ & $0.1 \mathrm{~s}$ \\
Maximized angle of rudder $\delta_{\max }$ & $30^{\circ}$ \\
\hline
\end{tabular}




\begin{tabular}{cc}
\hline Destructive radius $R_{\text {destroy }}$ & $10 \mathrm{~m}$ \\
Anticipant attack angle $\theta_{\text {expectaion }}$ & $-80^{\circ}$ \\
Effective distant of seeker $R_{\text {lock }}$ & $8.5 \mathrm{~km}$ \\
Maximized LOS angle of seeker $\phi_{\text {lock }}$ & $30^{\circ}$ \\
Estimated acceleration of target $a_{c}$ & $-0.4 \mathrm{~m} / \mathrm{s}^{2}$ \\
\hline
\end{tabular}

Table 2. Initial value of state

\begin{tabular}{cc}
\hline Denomination & Value \\
\hline Position X of leader $x_{L}$ & $20000 \mathrm{~m}$ \\
\hline Position Y of leader $y_{L}$ & $1000 \mathrm{~m}$ \\
Velocity of leader $v_{L}$ & $300 \mathrm{~m} / \mathrm{s}$ \\
Distance error of follower $\varepsilon_{R}$ & $300 \mathrm{~m}$ \\
Velocity of follower $v_{F}$ & $300 \mathrm{~m} / \mathrm{s}$ \\
Velocity of target $v_{T}$ & $50 \mathrm{~m} / \mathrm{s}$ \\
Others & 0
\end{tabular}

Base on above mentioned method and parameters, the results are provided as below and the flying curves of formation combat system are given at first.

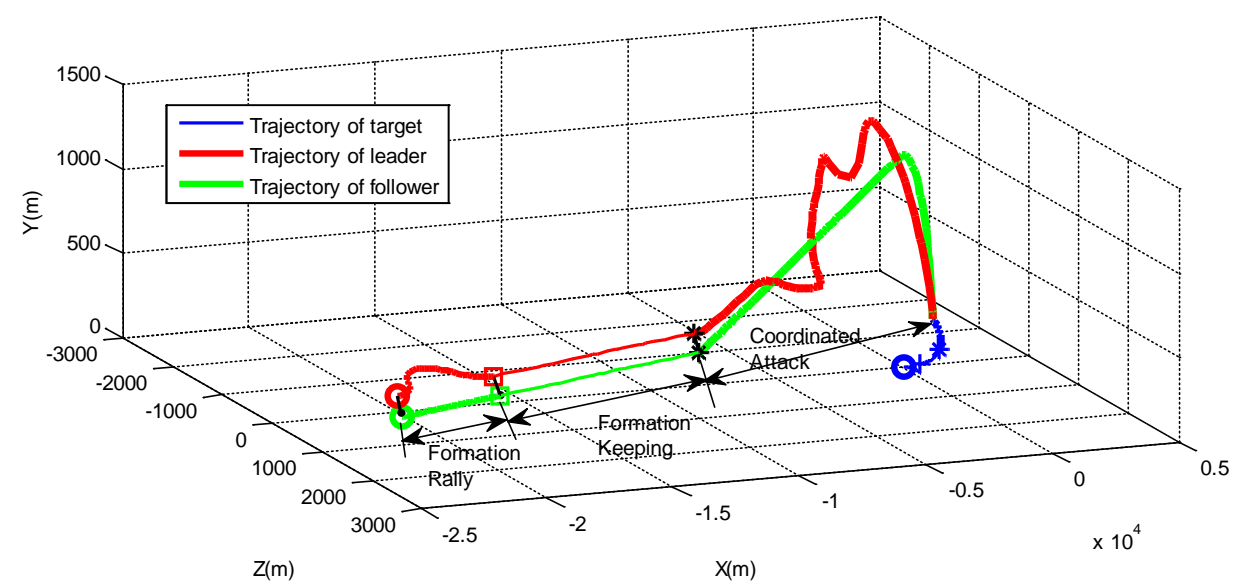

Fig. 4. Flying curve of formation combat system(3D)

In Fig. 4, o,+**are the origination point of three phase respectively; • is the anticipant position of follower. It is shown that the trajectories of formation members are continuous and smooth at the conterminous points, and reflect the mission characteristic of each phase clearly.

The key moments are:

$t_{r}=15.12, t_{\text {lock }}=48.86, t_{f}=85.4$; the distance between members and target is approximate to $10 \mathrm{~m}$ and the attack angle is $79.8^{\circ}$; the energy consumption is 84374.34 and can reduced by about $7 \%$ than the sum of consumption in three phases.

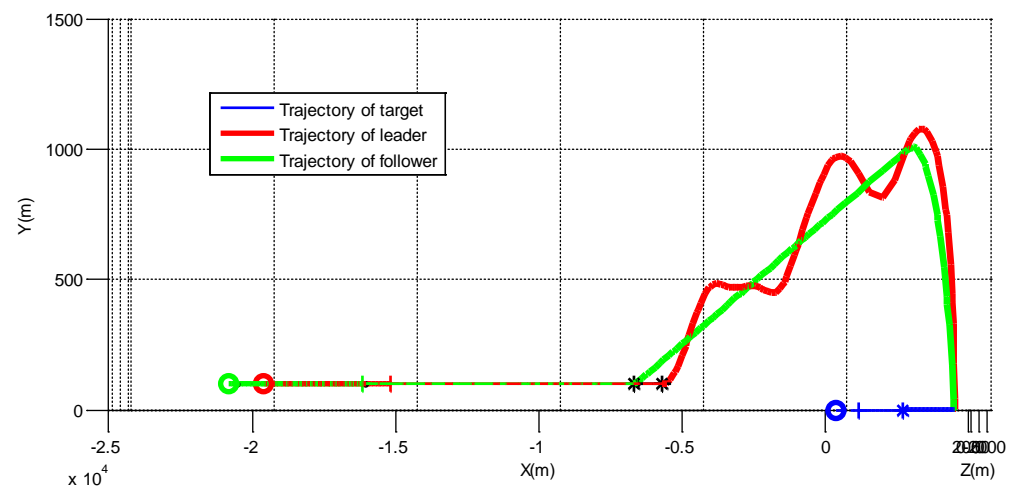

Fig. 5 Flying curve of formation combat system(Vertical) 
It is shown that the formation fly at horizontal in front of diving at the target in Fig. 5, because the maneuver in vertical section is limited in the formation rally phase and formation keeping phase artificially. It promotes the engineering with low coupling between pitch and yaw channels. In coordination attack phase, in order to attack the target with expected angle, the formation climbs to $1 \mathrm{~km}$ before swoop.

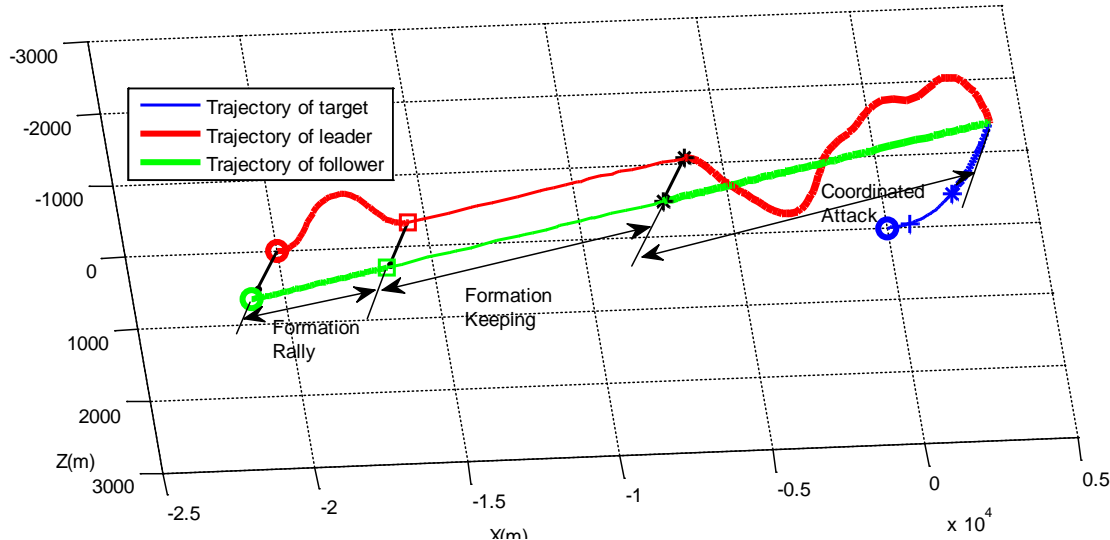

Fig. 6. Flying curve of formation combat system(Horizontal)

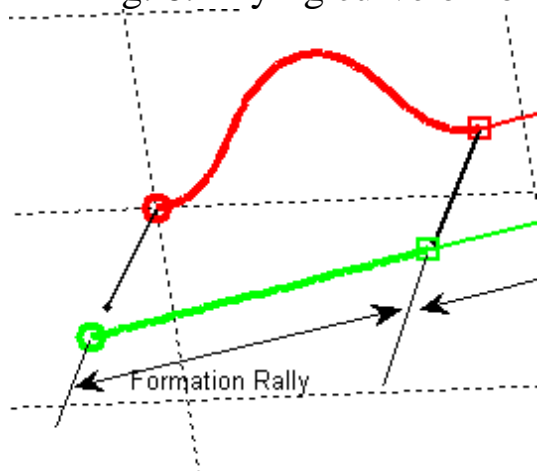

Fig. 7. Amplificatory local figure

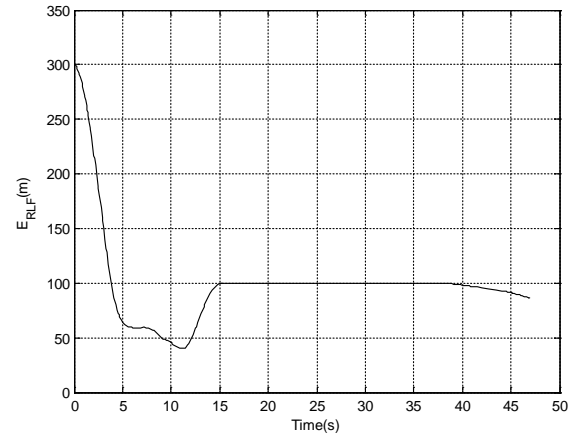

Fig. 8. Distance error of formation

The horizontal trajectories in Fig. 6 and Fig. 7 show that the follower flies straight to target for the sake of minimized consumption, when the leader maneuvers constantly in order to rally the formation and destroy target with the follower at the same time.

Second, variances of formation are shown in Fig. 8, Fig. 9 and Fig. 10. It is easy to see that requested accuracy of position and velocity is achieved in the formation rally phase and formation keeping phase.

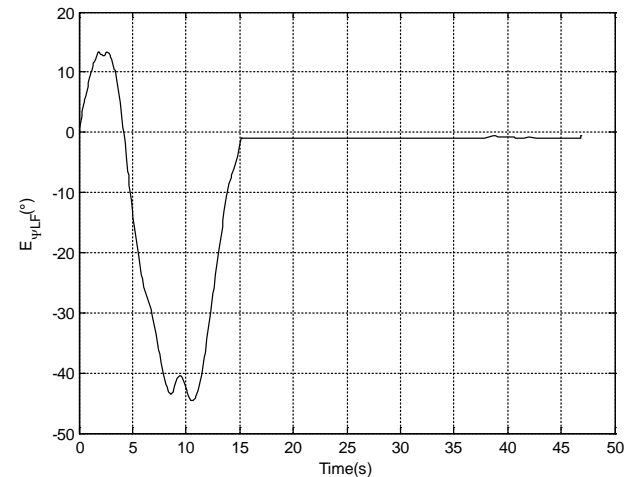

Fig. 9. Azimuth angle error of formation

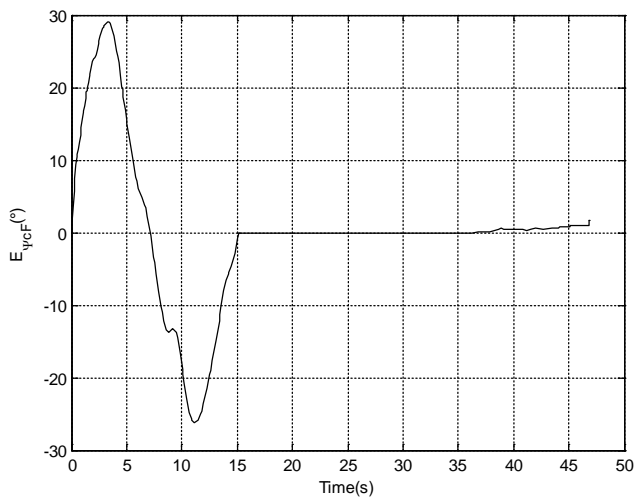

Fig. 10. Heading angle error of formation

In sum, the whole process of formation combat is satisfactory with instruction of optimal control designed by its integrative method, and all results have verified the validity of this integrative method. 


\section{Conclusions}

Key research aimed at the optimal control problem of formation combat in this paper is given as follows:

(1) The movement model is provided more actual with dynamic and control characteristics of formation members and maneuver characteristics of target.

(2) Optimal control models of each phase are built according to three subtasks, and the integrative optimal control model is put forward based on sub-models with additional two continuous restrictions.

(3) The simulation results with high precision and efficiency have verified the validity of multi-phase hp-adaptive Radau Pseudospectral Method which avoid choosing Legendre-Gauss-Radau points and carry out switch initiatively on three phases. It provides precondition for optimal online.

The integrative method can be used to formation combat with many members, and the estimated acceleration errors of target and static or dynamic threaten in formation combat system should be investigated.

\section{References}

[1]. Derek J. Bennet, M. Suzuki, K. Uchiyama. Autonomous Three-dimensional Formation Flight for A Swarm of Unmanned Aerial Vehicles. Journal of Guidance, Control, and Dynamics. Vol. 34( 2011), p.1899-1908.

[2]. Fang Liao, Rodney Teo, Jian Liang Wang, Kemao Peng. Robust Formation and Reconfiguration Control for Nonholonomic UAVs with Dynamic Constraints. AIAA Guidance, Navigation, and Control Conference, Boston, 2013, p.4629-4634.

[3]. Lee S., Park S. Y. Approximate Analytical Solutions to Optimal Reconfiguration Problems in Perturbed Satellite Relative Motion. Journal of Guidance, Control, and Dynamics. Vol.34(2011), p.1097-1110.

[4]. Scharf, Daniel P., Hadaegh Fred Y., Ploen Scott R. A Survey of Spacecraft Formation Flying Guidance and Control (Part II). NASA, 2006. Report No.20060043369.

[5]. WANG Xiang-ke, LI Xun, ZHENG Zhi-qiang. Survey of Developments on Multi-agent Formation Control Related Problems. Control and Decision, Vol.28(2013), p.1601-1614.

[6]. Desai J, Ostrowski J, Kumar V. Modeling and Control of Formations of Nonholonomic Mobile Robots. IEEE Trans on Robotics and Automation. Vol. 17(2001), p.905-908.

[7]. Chen J, Sun D, Yang J. Leader-follower Formation Control of Multiple Nonholonomic Mobile Robots Incorporating A Receding-horizon Scheme. Int J of Robotics Research, Vol.29(2010), p.727-747.

[8]. Arrichiello F, Chiaverini S, Indiveri G. The Null-space Based Behavioral Control For Mobile Robots with Velocity Actuator Saturations. Int J of Robotics Research, Vol.29(2010), p.1317-1337.

[9]. Kim S, Kim Y. Three Dimensional Optimum Controller for Multiple UAV Formation Flight Using Behavior-based Decentralized Approach. International Conference on Control, Automation and Systems, ICROS, 2007, p.1387-1392.

[10]. Lewis R, Tan K. High Precision Formation Control of Mobile Robots Using Virtual Structures. Autonomous Robots, Vol. 4(1997), p.387-403. 
[11]. Yoshioka C, Namerikawa T. Control of Nonholonomic Multi-vehicle Systems Based On Virtual Structure. Proc of the 17th Int Federation of Automatic Control World Congress, Seoul, 2008, p.5149-5154.

[12]. Desai J. A Graph Theoretic Approach for Modeling Mobile Robot Team Formations. J of Robotic Systems, Vol.19(2002), p.511-525.

[13]. Olfati-Saber R, Murray R. Graph Rigidity and Distributed Formation Stabilization of Multi-vehicle Systems. Proc of the IEEE Int Conf on Decision and Control, Las Vegas, 2002, p.2965-2971.

[14]. Keum W. Lee, Sahjendra N. Singh. Variable Structure Model Reference Adaptive Formation Control of Spacecraft. Journal of Guidance, Control, and Dynamics, Vol.35(2012), p.104-115.

[15]. Garcia-Taberner, Masdemont J. J. FEFF Methodology for Spacecraft Formation Reconfiguration in the Vicinity of Libration Points. Acta Astronautica, Vol. 67(2010), p.810-817.

[16]. Ashwini Ratnoo, Tal Shima.Formation-Flying Guidance for Cooperative Radar Deception. Journal of Guidance, Control, and Dynamics, Vol.35(2012), p.115-140.

[17]. I. S. Jeon. Impact time control guidance law for anti-ship missiles. IEEE Transactions on control systems technology, Vol.14(2006), p.16-25.

[18]. B. Jung, Y. Kim. Guidance Laws for Anti-Ship Missiles Using Impact Angle and Impact Time. AIAA Guidance, Navigation, and Control Conference and Exhibit, Keystone, Colorado, 2006, p.1-13.

[19]. Massari M., Bernelli-Zazzera F. Optimization of Low-thrust Reconfiguration Maneuvers for Spacecraft Flying in Formation. Journal of Guidance, Control, and Dynamics, Vol.32(2009), p.1629-1638.

[20]. Huntington G. T., Rao A. V. Optimal Reconfiguration of Spacecraft Formations Using the Gauss Pseudospectral Method. Journal of Guidance, Control, and Dynamics, Vol.31(2008), p.689-698.

[21]. Darby C L, Hager W W, Rao A V. An hp-adaptive Pseudospectal Method for Solving Optimal Control Problem. Optimal Control Applications and Methods, Vol. 32(2011), p. 476-502. 